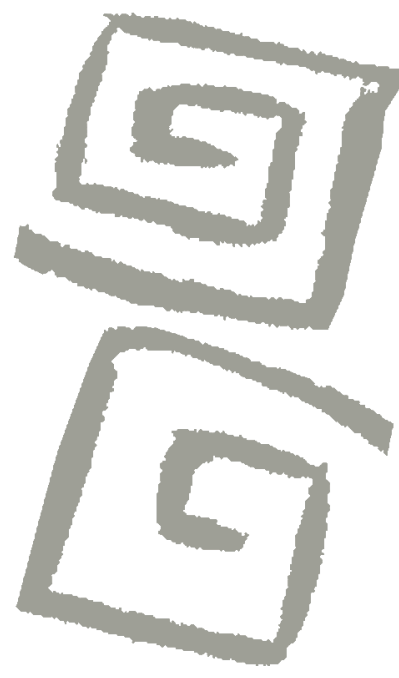

\title{
Desarrollo de un sistema georreferenciado para la gestión, movilidad y monitoreo de atención primaria de la salud comunitaria
}

\author{
Development of a georeferenced system for the \\ management, mobility and monitoring of primary care \\ in community health
}

Juan Manuel Diez Tetamanti ${ }^{1}$, Eduardo Rocha², Glauco Munsberg ${ }^{3}$, Jessica Helena Peixoto Castro ${ }^{4}$, Aline dos Santos Neutzling ${ }^{5}$, Sergio Fernando Jaime ${ }^{6}$, Leonardo Javier Schuler ${ }^{7}$

'Doctor en Geografía. Investigador, Consejo Nacional de Investigaciones Científicas y Técnicas. Docente Investigador, Grupo de Investigación Geografía, Acción y Territorio (GIGAT) Instituto de Investigaciones Geográficas de la Patagonia, Universidad Nacional de la Patagonia San Juan Bosco. Chubut, Argentina. $\triangle$ (iD)

${ }^{2}$ Doctor en Arquitectura Profesor, Universidade Federal de Pelotas.

Pesquisador, Programa de Pós-graduação, Faculdade de Arquitetura e Urbanismo, Universidade Federal de Pelotas. Pelotas, Brasil. $\bowtie$ iD

${ }^{3}$ Maestrando en Ciência da Computação, Universidade Federal de Pelotas. Pelotas, Brasil. $\triangle$ (iD)

${ }^{4}$ Arquitecta, Urbanista. Becaria, Coordenação de Aperfeiçoamento de Pessoal de Nivel Superior. Pelotas, Brasil. $\triangle$ (iD)

${ }^{5}$ Doctora en Ciencias Agrícolas. Universidade Federal de Pelotas. Pelotas, Brasil. $\triangle$ iD

${ }^{6}$ Médico, Magíster en Salud Pública. Hospital Zonal Trelew, Chubut, Argentina. $\triangle$ iD

Estudiante de Licenciatura en Geografía, Universidad Nacional de la Patagonia San Juan Bosco. Becario, Secretaría de Políticas Universitarias, Chubut, Argentina. $\square$ (iD
RESUMEN Los agentes de salud comunitaria en terreno practican acciones de fuerte impronta territorial, tanto en relación con prevención y asistencia, como a partir de un acercamiento de los servicios de salud a las familias. Estas prácticas en terreno, construyen un permanente mapeo colectivo basado en el conocimiento que los agentes poseen del territorio de actuación. A partir de un intenso trabajo de campo iniciado en el año 2012, en conjunto con organizaciones sociales locales e instituciones de salud de Brasil y Argentina, se inició el proyecto binacional App + Salud, que tiene como objetivo el desarrollo de un sistema de monitoreo y georreferencia para la gestión de la salud comunitaria, en formato de aplicación, para celular o tablet y acceso web, que considere la condiciones básicas, de ambiente y movilidad histórica de los usuarios, y que vincule metodologías de actualización cartográfica a partir de cartografía social con plataformas de mapeos colaborativos libres. Como resultado, se ha obtenido el desarrollo del sistema App + Salud, en nivel beta, para lo cual se profundizó en un trabajo de intercambio de saberes interdisciplinares.

PALABRAS CLAVES Servicios de Salud Comunitaria; Sistemas de Información Geográfica; Desarrollo Tecnológico; Argentina; Brasil.

\begin{abstract}
Community health agents carry out significant actions on the ground to provide prevention and care and bring health services to families. These practices in the field constitute a constant collective mapping process using the knowledge of the territory that the agents possess. Based on intensive fieldwork starting in 2012 in conjunction with local social organizations and health institutions in Brazil and Argentina, the binational project App + Health (App + Salud in Spanish and App+Saúde in Portugués) was initiated. The project's objective was to develop a monitoring and georeferencing system for community health management in the form of an application for cell phones or tablets with internet access, which would take into account the basic conditions of the environment and movement history of users and connect map updating methodologies using social cartography with free collaborative mapping platforms. As a result, the beta version of $\mathrm{App}+$ Health was developed, through a heightened process of exchange of interdisciplinary knowledge.
\end{abstract}

KEY WORDS Community Health Services; Geographic Information Systems; Technological Development; Argentina; Brazil. 


\section{INTRODUCCIÓN}

En el año 2012, el Grupo de Investigación Geografía Acción y Territorio (GIGAT) del Instituto de Investigaciones Geográficas de la Patagonia (IGEOPAT) de la Universidad Nacional de la Patagonia San Juan Bosco comenzó a trabajar en conjunto con la Tecnicatura Superior en Salud Comunitaria, de la provincia de Chubut, para cooperar con estudiantes y graduados en dos procesos: a) el análisis sobre las prácticas de accesibilidad a la salud en áreas rurales y; b) la elaboración de metodologías colectivas que cooperen en la práctica en terreno de los técnicos en salud comunitaria.

La práctica de los agentes de salud comunitaria genera acciones de anclaje territorial, las cuales pueden clasificarse en acciones de promoción y prevención de la salud, recopilación de datos de relevancia sociosanitaria en terreno, implementación de programas y planes nacionales y provinciales en escala comunitaria, y el afianzamiento de vínculos con las poblaciones de las zonas de influencia de los centros de atención primaria a los cuales se referencian ${ }^{(1)}$; todas estas acciones tienen como objetivo mejorar la salud de las personas a través de un acceso y distribución equitativa a las prestaciones de salud, desde una perspectiva del derecho a una protección integral de la salud ${ }^{(2)}$.

Dentro de las acciones orientadas a la recopilación de datos sociosanitarios, los agentes de salud comunitaria ejercen su tarea cotidiana realizando visitas regulares a las familias alcanzadas por una serie de programas sociales y sanitarios. Estas tareas de visita sistemática, denominadas rondas, proveen al sistema de salud de una serie de datos referidos al ambiente, el individuo y el hogar. Actualmente esa información es recopilada en formato papel, con un bajo nivel y factibilidad de sistematización y cruce de datos, por lo que presentan limitaciones para su análisis y utilización en el diseño de intervenciones que se propongan un cambio en las prestaciones de salud de las poblaciones implicadas.
En este marco, el trabajo desde el GIGAT se inició con el objetivo de realizar talleres de cartografía social a partir de la metodología abordada por nuestro grupo desde $2011^{(3,4)}$ destinada a agentes de salud comunitaria en diferentes localidades de la provincia de Chubut, en los que se desarrollaron diversos dispositivos metodológicos para el abordaje de problemáticas como: accesibilidad, prácticas cotidianas, relevamientos territoriales, etc.

Los talleres de cartografía social derivaron en la necesidad de hacer una revisión de las prácticas en función de la utilización posterior de los datos, sus posibles sistematizaciones y reutilizaciones en un marco histórico de seguimiento para cada familia y/o individuo. En la revisión de la práctica, se analizaron tanto la metodología de visita, como los mapeos que utilizan los técnicos en salud comunitaria, los métodos de rondas y los sistemas de recolección de datos (denominados planillas de familia). Así, en 2013, se elaboró una base de datos vinculada a un sistema de información georreferenciada, que permitía transferir los datos en papel, a un formato digital y georreferenciado.

A partir de este trabajo, se inició un proceso en conjunto con el Laboratorio de Urbanismo (LABurb), la Faculdade de Arquitetura de la Universidade Federal de Pelota (UFPel), en Brasil, con quienes se comenzó a pensar los primeros pasos posibles para integrar estas soluciones al levantamiento de datos, sistematización e integración de metodologías, en un sistema complejo y georreferenciado, que facilite el trabajo de los agentes de salud comunitaria y permita agilizar el manejo de datos, hacer vinculaciones y visualizaciones complejas, al tiempo que se recupere la textura social territorial.

El sistema fue bautizado por ambos grupos de trabajo como App+Salud (App+Saúde, en portugués) y actualmente se encuentra en una etapa de testeo y desarrollo, disponible en dos versiones: aplicación para smartphone (para android) y web abierta al público para su prueba (http://www.appsalud.unp.edu.ar).

El objetivo de este desarrollo se concentra en crear un sistema georreferenciado de monitoreo que organice la información 
sociosanitaria local, fragmentada en células familiares e individuales y que, al mismo tiempo, permita un análisis integral con las singularidades del territorio en el que esas familias e individuos habitan.

El sistema se organiza en tres grandes componentes: a) un sistema georreferenciado de monitoreo que organiza los datos sanitarios del lugar y facilita consultas de situación, prevención y pronóstico de eventos; b) la aplicación para smartphone de uso restricto a los agentes de salud y otro abierto a la comunidad, que permita mejorar los datos geográficos para monitoreo $y ; c)$ un dispositivo metodológico de traducción de cartografía social, que incorpore elementos sociales, culturales, temporales y coyunturales en el mapeo general (como basurales, estado de cursos de agua, presencia recurrente de humo, etc.).

Para el desarrollo de la aplicación, actualmente en proceso de testeo, se analizaron las prácticas, metodologías e instrumentos de trabajo en terreno de los agentes de salud comunitaria, las singularidades de acceso a la salud en áreas rurales y urbanas vulnerables y los tipos de mapeo desarallados por los agentes de salud.

El texto de este artículo pretende dar cuenta del proceso abordado (que aún no ha finalizado), por un grupo multidisciplinario integrado por geógrafos, médicos, arquitectos, técnicos en salud comunitaria, programadores de informática, comunicadores sociales y estudiantes, entre otros. Las conclusiones y resultados son de carácter parcial, dadas las características propias del proceso de construcción del proyecto continuo y los enfoques a los cuales adherimos desde la fenomenología y la cartografía ${ }^{(5,6)}$.

\section{El enfoque inicial, experiencias y producción}

La experiencia territorial, a partir de la práctica de salud comunitaria, impulsa el diálogo como productor de conocimientos que derivan en otros múltiples territorios y singularidades, como ingredientes para nuevas prácticas en el trabajo cotidiano. En este sentido, vamos a iniciar esta argumentación sobre la convicción de que no hay "un solo territorio" a abordar sino que, como construcción, es múltiple, variado y heterogéneo, con fuertes impresiones por las características sociales locales, que lo hacen complejo $^{(7)}$ cuando intentamos generar representaciones homogeneizadas ${ }^{(8)}$.

Inicialmente, nos enfocamos en la perspectiva de la experiencia como método, en la cual el espacio se expresa como un aprendizaje $y$, al mismo tiempo, un como producto social colectivizado. Decimos que es colectivizado, dado que entendemos que la perspectiva experiencial no solo enfoca una realidad conocida a partir de la experiencia, es decir, de la comunión de pensamiento y sentimiento individual, como expone $\mathrm{Yi}-\mathrm{Fu}$ Tuan $^{(9)}$, sino que además, entendemos al sujeto y al objeto social unidos en esa comunión. Se amalgama así, tal como expone Kastrup, el proceso de la experiencia compartida y móvil entre los sujetos participantes del proceso de investigación y producción ${ }^{(10)}$. Este es en sí mismo un proceso de experiencia, que coloca tanto al investigador como el objeto de la investigación social en un plano común, en el que la experiencia es el proceso que los unifica y propicia al diálogo e intercambio de información, por el cual ambos, sujeto investigador y sujeto investigado (tradicionalmente, objeto), comparten elementos de la experiencia. Se produce así un proceso de enriquecimiento sobre el conocimiento de la realidad experimentada, a partir del diálogo de sujetos heterogéneos, que comparten sucesos comunes a través de diferentes subjetividades ${ }^{(9)}$.

En la práctica de los agentes de salud comunitaria, el espacio se evidencia como una obra comunitaria en permanente construcción, en la que la producción colectiva de la práctica sanitaria sobre las diversas realidades abordadas en cada hogar, cada familia, cada barrio, cada localidad avanza sobre nuevas posibilidades de intervención y abordaje. Las diversas singularidades, ambientales, sociales, vinculares, que identifican estos espacios de intervención, puestas 
en diálogo entre sí, se tornan de inmediato un escenario factible a ser abordado con diversos métodos y disciplinas, que también entran en diálogo pero que al mismo tiempo constituyen al territorio como un relato ${ }^{(11)}$.

Dentro del proceso de desarrollo de la App + Salud resulta pertinente citar dos artículos que discuten las nociones de territorio, espacio y programa. En primer lugar, nos interesa el texto de Breilh ${ }^{(12)}$, que propone la posibilidad de abordar una reflexión emancipadora sobre los problemas de salud en el territorio, con el propósito de "superar las restricciones clásicas de la teoría del espacio urbano y las concepciones reduccionistas sobre la salud y el ambiente, para acercarse a la necesidad colectiva"(12). En este sentido, Breilh indica la necesidad de un abordaje que implique el proceso histórico del espacio social, como parte de la construcción de una multiplicidad de singularidades que conforman "modos de vida colectivos". Así, el autor enfatiza la relación entre la epidemiología crítica y la geografía crítica como alternativa para el abordaje de una mirada que vaya más allá de la perspectiva empírica reduccionista del lugar, anclada desde la perspectiva euclidiana del "espacio como receptáculo". En esta línea, Breilh indica que no hay un espacio urbano de la salud como lugar estático, continente y pasivo, sino un espacio siempre complejo y en movimiento creador $^{(12)}$. Desde este enfoque, coincidimos en la posibilidad de abordar -tanto desde la cartografía social como desde el desarrollo de aplicaciones tecnológicas interactivas que rescaten la textura social y ambiental- herramientas que faciliten los procesos de sistematización y gestión integral de la información territorial, con una perspectiva de transformación comunitaria integrada.

Por otro lado, Spinelli ${ }^{(13)}$ advierte que el territorio invita a generar nuevas preguntas, por lo que en la práctica territorial, "enfrentar lo no pensado, y aceptar el derrumbe de las certezas, permitirá realizar preguntas y evitar la naturalización, la queja y/o la anécdota"(13). Así, Spinelli avanza sobre conceptos de la geografía crítica y la teoría miltonsantiana en función del territorio como elemento inacabado y como calidoscopio que se complejiza en lo micro. También enmarca lo territorial desde la idea de rizoma y en el sentido deleuziano, por lo que indica que en:

...el proceso de cartografiar no hay protocolos posibles ya que su práctica es netamente micropolítica y singular. Ante ello, solo debe regir el imperativo de intervenir, de jugar, de desencadenar procesos sin certezas, pero fiel al deseo y a las apuestas que se generan, tratando que las acciones sean el puente entre la potencia y el poder. ${ }^{(13)}$

En este sentido, adherimos a la construcción de nuestro método de trabajo y producción de la aplicación, que parte de la práctica cotidiana del agente comunitario en la construcción de formularios participativos, la microcartografía de relevamientos en los centros de salud y los talleres de cartografía social, con plena base fenomenológica. Por esto, adherimos plenamente a la postura de Spinelli cuando indica que

...es necesario entender que no hay un método ni una técnica para intervenir en el juego social del territorio. Este texto expresa el esfuerzo por salir de los modelos racionalistas que dominan las formas de trabajar y de pensar lo social, para pasar a dinámicas marcadas por el juego, el devenir y la incertidumbre que son propias de lo social. ${ }^{(13)}$

Desde el georreferenciamiento, existen múltiples trabajos que pueden citarse, tanto en Argentina, como en el exterior. Una de las últimas producciones de Buzai, "Análisis espacial en Geografía de la Salud ${ }^{(14) "}$ aporta una rica batería de herramientas y procedimientos para el tratamiento y análisis de datos en salud, bajo un enfoque analítico territorial. También otros trabajos como los de Barcellos et al.(15) e Hino et al. ${ }^{(16)}$ ejemplifican posibilidades de aplicación de técnicas de geoproceso y metodologías de estudio con enfoque analítico. No obstante, los trabajos de Muller, Cubas y Bastos ${ }^{(17)}$ y Da Fonseca ${ }^{(18)}$ 
hacen hincapié en la construcción de una metodología que involucre el saber de los agentes sanitarios en la propia producción de esquemas de análisis.

El proyecto App+Salud puede enmarcarse dentro de lo que la Organización Mundial de la Salud (OMS) denomina mHealt (abreviatura de mobile health), que incluye todas las iniciativas de desarrollo que involucran dispositivos móviles para la gestión, geoprocesamiento, control, comunicación y monitoreo de casos e intercambio de información sanitaria. En este marco, se destaca el proyecto Commcare ${ }^{(19)}$, con actual aplicación en el sudeste africano por parte de múltiples ONG, organismos internacionales y gobiernos locales $20,21,22)$.

Así, el desarrollode App + Salud, no puede suscribirse simplemente al desarrollo de una aplicación informática para dar solución a un proceso de sistematización y georreferenciación. El desarrollo de App+Salud es en sí mismo un proceso de experiencia implicada y compartida entre sujetos diferentes, lugares diferentes, prácticas diferentes y profesiones diferentes. En esencia, desde una perspectiva deleuziana, es la diferencia puesta en diálogo y sus fuerzas en resistencia, que se pone a producir elementos nuevos, a partir de su propia complejidad rica en sí misma, desde la multiplicidad ${ }^{(23)}$.

\section{MÉTODO Y PRIMERAS EXPERIENCIAS EN ÁREAS RURALES}

\section{Cartografía Social y SIG, con agentes de Salud Comunitaria en Argentina}

A partir de la relación de trabajo en conjunto entre el GIGAT y la Tecnicatura Superior en Salud Comunitaria y el desarrollo de talleres de cartografía social -proceso en el que se filmó el video Ensayos de Cartografía Social en Patagonia(24) - en 2013, se desplegó un dispositivo de investigación e intervención en el que se abordaron las localidades de Alto Río Senguer y Aldea Beleiro (Chubut, Argentina). El dispositivo de trabajo $^{(25)}$ de una semana en cada localidad, se construyó íntegramente con referentes locales y provinciales de salud y se enfocó sobre la pregunta “¿cuáles son las estrategias locales de la población y de los agentes de salud para garantizar el acceso general a la atención sanitaria?". En esta línea, la experiencia de los agentes en salud comunitaria se posicionó en un lugar central, a partir de su relación con la población local, en tanto práctica cotidiana.

La metodología de trabajo se configuró en un dispositivo de investigación, detallado en Chanampa et al. ${ }^{(26)}$, el cual se diseñó en dos grandes esferas:

a. Indagación sobre las prácticas locales sanitarias y la relación de la atención primaria de la salud con la comunidad; realizado a partir de talleres de cartografía social, para el análisis de la accesibilidad a la salud, estrategias de movilidad, facilidades y problemas de acceso a la salud.

b.Procesamiento de georreferenciación de las familias asistidas por los agentes de salud comunitaria, en el que se sistematizó la información recopilada en las planillas de familia.

Para la esfera "a", se creó un dispositivo de trabajo de cinco días, cuya metodología consistió en visitar a las familias de las localidades, con la compañía de agentes de salud comunitaria. Se desarrollaron entrevistas a referentes y se planificó, en conjunto con los agentes de salud locales un taller de cartografía social destinado al análisis de la accesibilidad a la salud ${ }^{(13)}$. El taller se enfocó en aspectos como accesibilidad y estrategias, motivos y lugares de atención sanitaria, facilidades, obstáculos y movilidad en función de estrategias de la población local para el acceso a los servicios de salud.

En esta esfera se trabajó sobre la base de siete entrevistas semiestructuradas a referentes de la gestión de la salud, nacional, provincial y municipal, y agentes de salud comunitaria, realizadas de 2012 a 2015; cuatro talleres de cartografía social realizados entre 2013 y 2015 en las localidades de Comodoro Rivadavia, Aldea Beleiro, Río 
Senguer (Argentina) y Pelotas (Brasil) con la participación de agentes sanitarios y familias convocadas por los propios agentes, cada uno de los cuales contó con la participación de un promedio de 25 integrantes, divididos en grupos de cinco personas; y dos talleres de discusión sobre prácticas en visitas de familia con la participación de diez referentes de salud comunitaria: uno en Comodoro Rivadavia, en la Dirección del Área Programática Sur, en 2014, y otro en Pelotas en la Dirección de Salud Regional del distrito, en 2015.

Para la esfera " $b$ ", la metodología se enfocó en el diseño de una base de datos en un sistema de información georreferenciada, utilizando el programa de licencia libre
QGIS, para emular las planillas de familia, para luego realizar una sistematización de la información y el cruce de diversos datos. El proceso de georreferenciación de las planillas de familia, se realizó con el apoyo de una imagen satelital, cartas topográficas del Instituto Geográfico Nacional y el reconocimiento visual de un agente de salud, un baqueano, chofer de ambulancia.

A partir de esta georreferenciación de las planillas de familia se generaron los mapas de la Figura 1 y la Figura 2 como muestreo piloto del trabajo desarrollado, con el propósito de evaluar los beneficios de sistematizar y georreferenciar la información en conjunto con los agentes de salud locales.

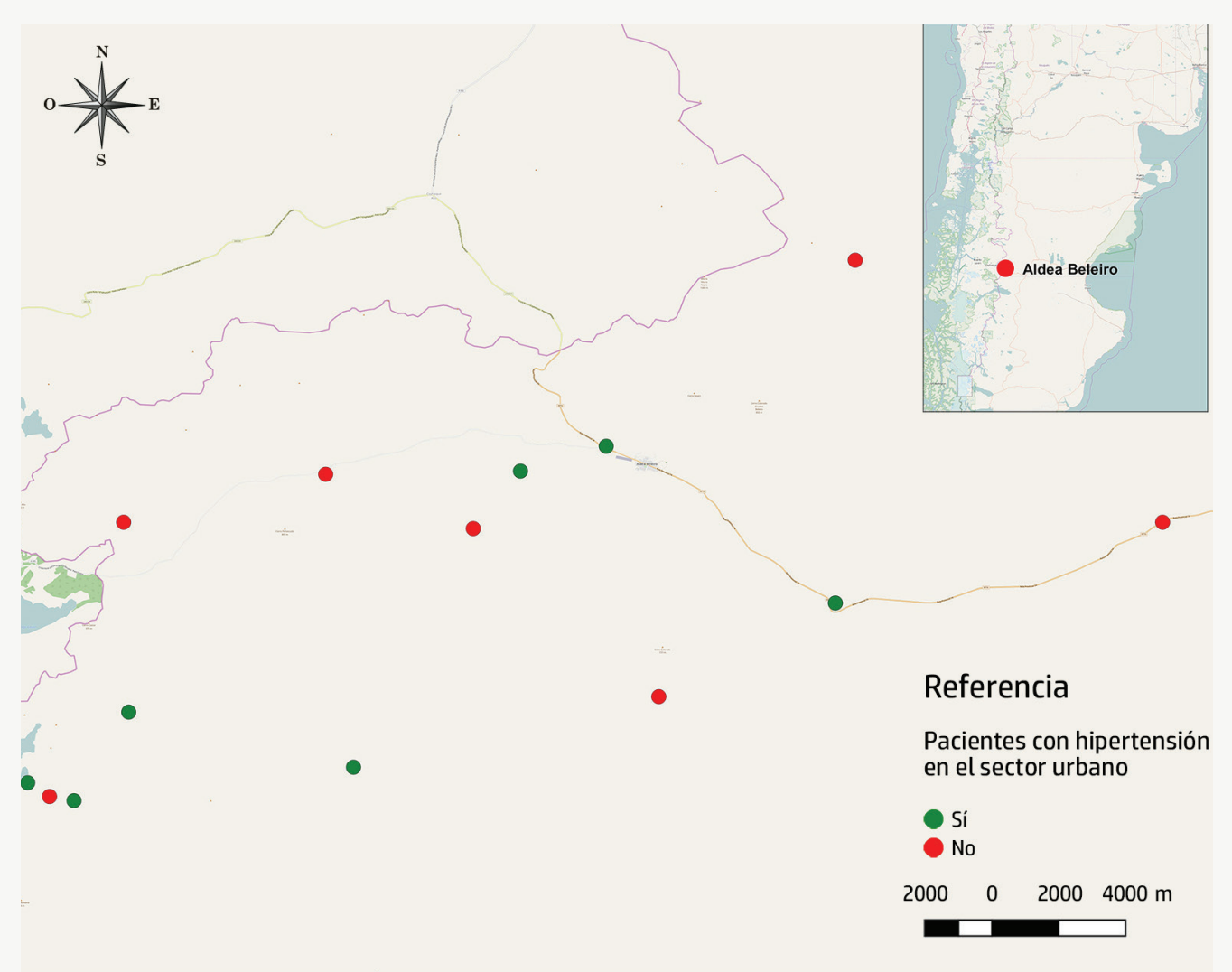

Figura 1. Distribución espacial de pacientes con hipertensión en el área Aldea Beleño. Mapeos generados a partir de la georreferenciación de datos de las planillas de familia en papel. Provincia de Chubut, Argentina, 2013.

Fuente: Planilla de familias relevadas por técnicos en salud comunitaria. Nota: Cartografía elaborada por Leonardo Javier Schuler. 
Finalmente, se sistematizó el trabajo realizado en los talleres de Cartografía Social, en tablas de resultados que permitieron establecer análisis críticos de accesibilidad a la salud en áreas rurales. Este mapeo social se constituyó en la primera traducción entre el proceso de construcción de Cartografía Social y su producción para la visualización en salidas cartográficas generadas a partir del programa QGIS.

El desarrollo de una serie de mapas permitió la incorporación de datos con contenido de conocimiento a partir de la experiencia social local (basurales, vados, sectores anegados, etc.) en objetos espaciales en la plataforma libre y colaborativa openstreetmaps. Esta adición de contenido se consideró relevante para socializar comunitariamente la información geográfica y generar abordajes críticos a las diferentes singularidades locales.

El proceso comentado hasta aquí, argumentó las bases de aportes para el desarrollo de la App+Salud, cuyos resultados resumimos en el Cuadro 1.

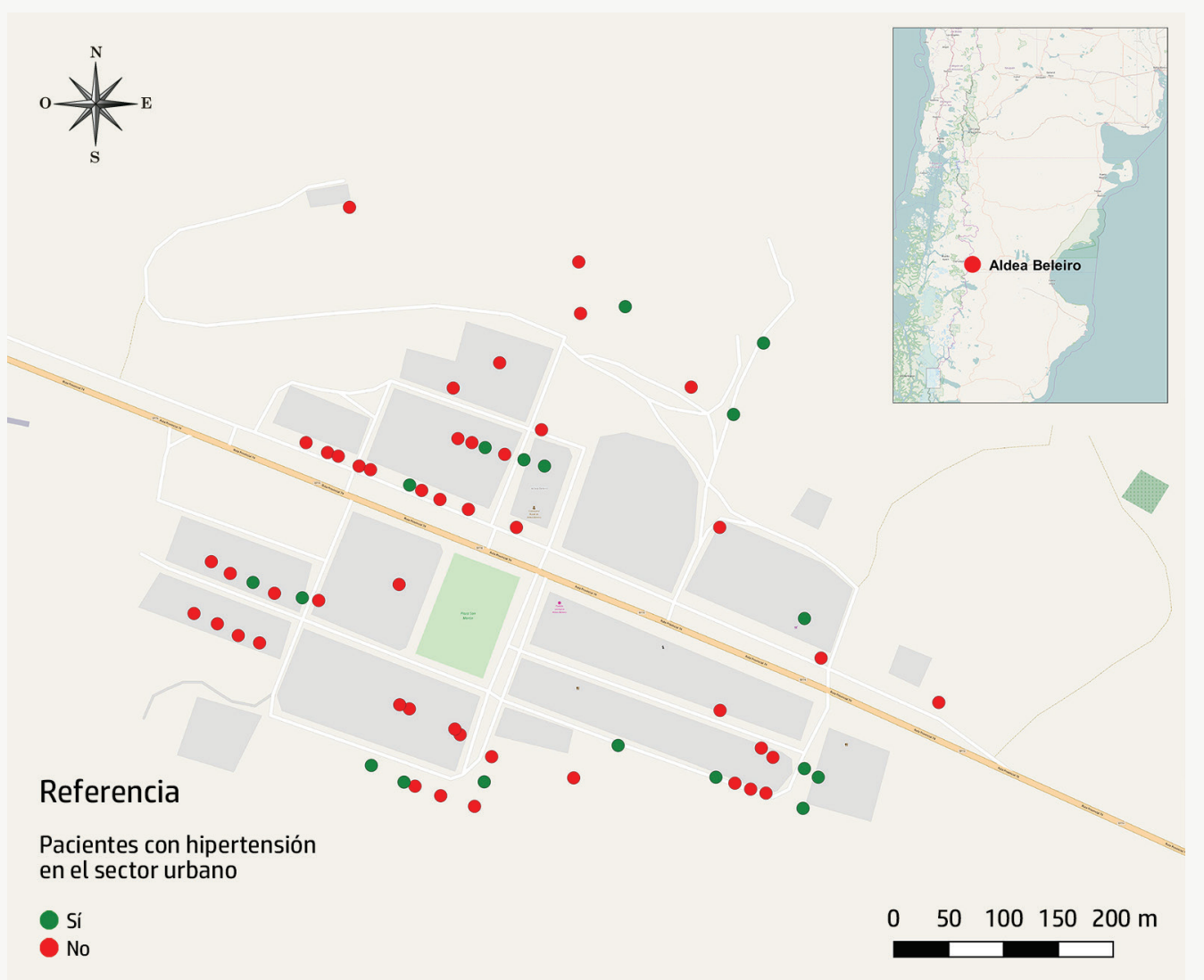

Figura 2. Distribución espacial de pacientes con hipertensión en el área Aldea Beleño. Mapeos generados a partir de la georreferenciación de datos de las planillas de familia en papel. Provincia de Chubut, Argentina, 2013.

Fuente: Planilla de familias relevadas por técnicos en salud comunitaria. Nota: Cartografía elaborada por Leonardo Javier Schuler. 
Cuadro 1. Aportes de la cartografía social, la sistematización y georreferencia de la información, y de la comunión de metodologías al desarrollo de la App+Salud.

Aportes de la cartografía social

- Recuperación de la textura social del espacio, en función de las problemáticas y estrategias desarrolladas por la población local.

- Apertura del diálogo local sobre la problemática de salud con enfoque territorial, intergeneracional y de redes.

- Comparación de estrategias en diferentes periodos temporales facilitadoras del diálogo intergeneracional (el ejemplo de las prácticas de parto y los diferentes lugares en donde se practicaban).

- Reformulación de límites, fronteras y nodos, en función de las prácticas sociales.

- Diálogo entre las formas y el contenido del espacio, a partir de la producción de nuevos mapeos colectivos.

- Incorporación de nuevos contenidos a las formas formales (como por ejemplo, zonas anegadas por nieve, ríos que impiden el paso por caminos en épocas del año, etc).

- Producción de otros mapas de proyección y escala social.
Aportes de la sistematización y georreferencia de la información

- Digitalización de la información, hasta el momento solo disponible en soporte papel

- Posibilidad de manejo, vinculación, relación, cruce y análisis de los datos para generar nueva información.

- Operaciones de traducción de mapas y cartas en papel a SIG, con la colaboración de baqueanos ${ }^{(27)}$.

- Georreferencia de la información, y unificación de los dispositivos de almacenamiento, y visualización, como facilitadores de la gestión de la salud comunitaria en terreno.

- Posibilidad de realizar superposiciones de información sanitaria y geográfica para el análisis de problemáticas locales y regionales.

- Posibilidad de realizar seguimientos históricos y sistemáticos.

- Se consideró como obstáculo la capacitación de los agentes de salud en manejo de SIG y posibles fragmentaciones en criterios de uso en cada localidad.

Aportes de la comunión de metodologías

- Actualización de los mapas base, particularmente utilizando la plataforma https://www.openstreetmap.org

- Incorporación de información obtenida a partir de Cartografía Social en la plataforma https://www.openstreetmap.org como mapa base (basurales informales, crecidas de arroyos temporales, áreas contaminadas, etc.).

- Creación de un shape con la información recopilada en la planilla de familia.

- Superposición de capas y análisis integral de la información obtenida en ambos procesos metodológicos.

Fuente: Proyecto App+Salud

\section{Sensibilización y profundización de experiencias en trabajo de campo, camino al diseño de App + Salud}

Con todo el proceso transitado hasta aquí, se inició una etapa de intercambio de la experiencia con integrantes del LabUrb de la Universidade Federal de Pelotas, a partir del cual se comenzó a redactar un proyecto binacional que, en 2014, obtuvo financiación del Conselho Nacional de Desenvolvimento Científico e Tecnológico de Brasil y, en 2015, de la Secretaría de Políticas Universitarias de Argentina. Así se inició un trabajo de investigación-intervención en talleres de sensibilización con agentes de salud comunitaria y funcionarios públicos de Salud (en Argentina, con el Ministerio de Salud de la provincia de Chubut y del municipio de Comodoro Rivadavia; en Brasil, con funcionarios del
Ministerio da Saúde del estado de Río Grande do Sul y la prefectura de Pelotas). Este trabajo tuvo un doble propósito: a) experimentar y socializar con los grupos de investigación el sistema actual de visitas a familias y levantamiento de datos en ambos países y; b) establecer pautas de traducción del sistema actual (en papel), a formatos digitales y georreferenciados que permitan no perder la textura social de la información.

Se realizaron dos talleres con los agentes de salud comunitaria en Argentina: uno en Comodoro Rivadavia y otro en Río Mayo sobre autoanálisis de prácticas de trabajo en terreno, con la participación de quince agentes de salud comunitaria en terreno y funcionarios provinciales. Estos talleres se organizaron con la participación de investigadores y estudiantes de ambos países y se obtuvieron los siguientes resultados críticos: 
a) superposición de agentes sociales en las visitas de familia, con réplica de información; b) críticas y sugerencias a la estructura planillas de familia (principal instrumento de los agentes de salud comunitaria para el registro y levantamiento de datos regular) y; c) análisis de los diferentes modelos de georreferenciación y mapeo utilizados por los agentes.

Con relación al punto vinculado a la superposición de agentes sociales, los resultados surgieron a partir de la elaboración de un sociodrama ${ }^{(28)}$ en el que los agentes realizaron simulaciones de las prácticas de visita de familia, en las cuales utilizan las planillas de familia y establecen múltiples vínculos sociales con las familias visitadas que habilitan la práctica de intervención social ${ }^{(29)}$. En este sentido, las planillas de familia, como "objeto" de seguimiento, se configuran en un instrumento más que, en ocasiones, pierde sentido de uso en un contexto en el que las connotaciones simbólicas de las prácticas sociales de salud ingresan por otras vías de registro y seguimiento. En este sentido, coincidimos con la posición de Carballeda, respecto de la práctica de intervención:

...la influencia del pensamiento médico y biológico, también Ileva a la intervención social a prácticas normalizadoras, moralizantes, punitivas y pedagógicas que se contradicen con los aspectos históricos sociales que atraviesan el proceso de intervención social y especialmente a la demanda. De este modo, pensar la Intervención en lo Social como dispositivo, supone un diálogo que abarque diferentes perspectivas de visibilidad, enunciación, surcos de poder, y especialmente de las formas de construcción de subjetividad que se liga a esta. Una manera de aproximación a este tema puede construirse desde la perspectiva lingüística y la construcción de subjetividad. ${ }^{(30)}$

Así, el sociodrama se inscribió como posibilidad de conocer y experimentar la práctica como contenido que nos permite no solo simular las visitas y representar sus complejidades, sino producir un escenario de intervención factible de adaptar en el desarrollo del proyecto App + Salud. Una adaptación en línea con modos de aproximación, en los que la normalización no opaque los aspectos sociales que atraviesa el proceso de intervención social.

Respecto del punto vinculado a las críticas y sugerencias a la estructura planillas de familia, a partir de entrevistas realizadas a supervisores y agentes de salud comunitaria en terreno, que participaron activamente en diversas modificaciones realizadas en los últimos cinco años a las planillas de familia, se realizaron revisiones de las diferentes versiones utilizadas como un instrumento que permite una compilación histórica de variables sociosanitarias, las cuales fueron consideradas como valiosas para su relevamiento en terreno y se aprecian como un instrumento fundamental en la práctica del trabajo comunitario. Dado su carácter histórico, estas planillas fueron incorporando variables a medida que cambiaba la percepción y el encuadre de los problemas de salud y las prioridades de abordaje. Como respuesta a la necesidad de ordenar la acumulación de datos y un manejo uniforme, en la provincia de Chubut, la Dirección de Promoción y Prevención publicó las Normas Provinciales del Trabajo en Terreno, que reglamentan los distintos aspectos del trabajo en terreno, incluyendo los contenidos de la planilla de familia que genéricamente se empezó a denominar "F1" y que se constituyó en la referencia obligada para su análisis. Esa planilla, en su versión 2015, es la que en un proceso conjunto entre el GIGAT, el LabUrb, los agentes de salud comunitaria y las autoridades del Ministerio de Salud, validaron en una serie de variables de igual contenido a las del "F1", pero en ocasiones adaptadas en sus categorizaciones, con el propósito de hacerlas amigables a lo que sería el futuro entorno digital para dispositivos móviles. Dentro de este análisis se reflexionó sobre la pertinencia y utilidad de variables repetitivas o redundantes, generando una depuración de la planilla de familia, con el objetivo de avanzar en una adaptación al formato digital. 
Luego de los trabajos de implicación, en la etapa de sensibilización, revisión y comparaciones, se indagó sobre los mapeos locales construidos por los agentes de salud comunitaria en terreno, tanto en puestos sanitarios rurales como en centros de salud barriales. En cada centro y/o puesto de salud visitado, encontramos diferentes y originales tipos de mapas utilizados para realizar una georreferenciación colectivizada de las familias atendidas en el área. Los mapas, que constituyen parte del archivo fotográfico del grupo binacional de investigación, fueron analizados y comparados en función de establecer recurrencias y diferencias en las existencias. Como particularidad, los mapas son generalmente realizados a mano (Figura $3)$, a veces calcando planos catastrales e incluyendo referencias de notable relevancia local como ríos, basurales, sectores de difícil acceso, etc. A su vez, los mapas tienen referenciadas las casas de familia -generalmente con un número propio- y situaciones particulares que las identifican (color de las paredes, nombre o sobrenombre de alguno de los habitantes, "vecino de", etc.). Mediante el número de identificación, cada mapa se vincula con los datos una planilla de familia, aunque este dato es de uso generalmente interno, circunscripto a la utilización del agente de salud y en ocasiones a un grupo restringido de compañeros de trabajo.

El desarrollo de esta etapa de sensibilización fue fundamental para el inicio del trabajo en conjunto con el programador de informática radicado en el LabUrb, quien debía realizar un profundo trabajo de "traducción" de los resultados obtenidos hasta el momento y las demandas de los agentes de salud comunitaria, en función del desarrollo base de la App + Salud.

\section{Articulación y adaptación de las planillas de levantamiento de datos}

El desarrollo inicial de la App + Salud requirió de tres reuniones de los equipos de ambos países en conjunto con el programador informático. Como medida previa al diseño de la estructura básica de la App + Salud, se analizaron los formularios utilizados por los agentes de salud, tanto en la provincia de Chubut, como en el municipio de Comodoro Rivadavia, la prefectura de Pelotas y el estado de Río Grande do Sul. Para el trabajo de integración de las diferentes planillas, desde el LabUrb también fue preciso organizar reuniones y entrevistas con secretarios, jefes de programas y

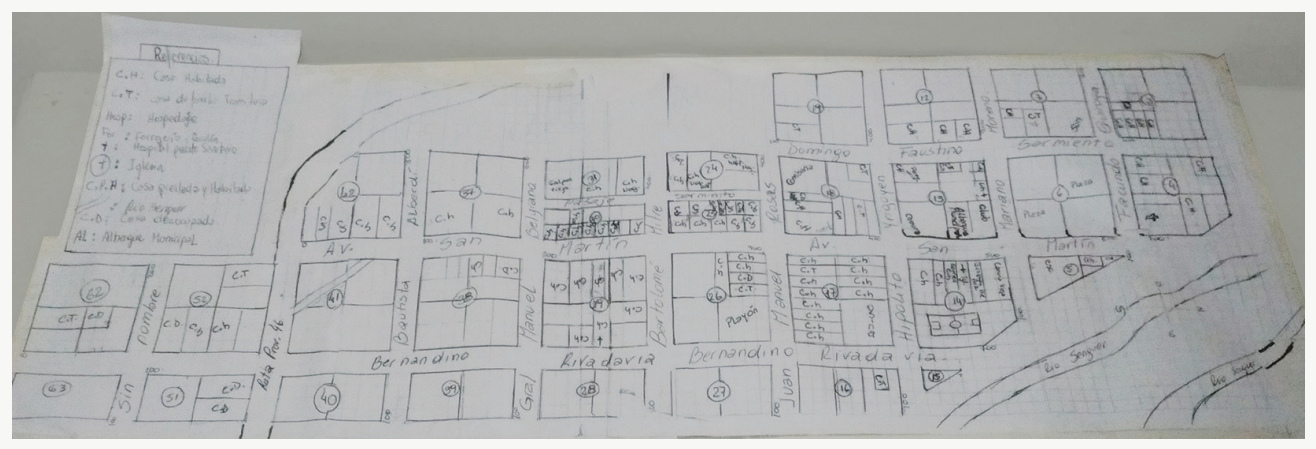

Figura 3. Típico mapa realizado en puesto sanitarios rurales, vinculado a Planillas de Familia. Provincia de Chubut, Argentina, 2014. 
directores del área de salud de la prefectura de Pelotas y el estado de Río Grande do Sul, con una metodología similar a la aplicada en Argentina, aunque con una impronta de importación de la propuesta generada inicialmente en Chubut.

Luego del análisis, los tipos de planillas utilizadas en Argentina (de Familia) y en Brasil (Sistema Único de Saúde - Atençao Básica, Programa Nacional de Controle da Dengue, Programa de Controle da Doença de Chagas, y programas municipales); se generó una tabla de contenidos de las planillas y sus singularidades, tipos posibles de integración y articulación y se diseñó un primer esquema de 210 preguntas con aspectos ambientales, de vivienda, programas sociales, personales y familiares. Este diseño incluía las singularidades de consultas que se realizan tanto en el área de trabajo argentino como brasileño articulando los tipos de planilla mencionados en un solo sistema de cuestionario.

\section{RESULTADOS}

\section{Desarrollo de App + Salud (App + Saúde) e integración de opensetreetmaps}

A partir del diseño del primer esquema de 210 preguntas, se pasó a una etapa de depuración y simplificación del cuestionario, en la que se realizaron talleres $\operatorname{críticos}^{(31)}$, en conjunto con agentes de salud comunitaria, tanto en Argentina como en Brasil para la mejora de la calidad del cuestionario.

Pasada esta etapa de depuración se logró un esquema de 177 preguntas incluyendo todos los aspectos temáticos que contienen la diversidad de planillas y formularios, y la georreferenciación de cada serie de datos, a partir del ingreso de una "visita a vivienda" que es la acción de acceso del agente de salud comunitaria a cada vivienda asignada. Dada la adaptación de las diversas planillas que se incluyen en la interrelación binacional de su génesis, la App + Salud está articulada para ingresar una visita a vivienda, desde la cual se incluyen los datos, aspectos ambientales y se listan los individuos que viven allí. A su vez, cada género o grupo etario está dividido en diferentes tipos de preguntas, con referencia a la singularidad del ingreso de cada nuevo individuo. Así, por ejemplo, las preguntas para una mujer embarazada son diferentes que las destinadas a otras mujeres que no estén en el periodo de embarazo. No obstante, estos datos quedan guardados en el "histórico" del individuo, que funciona como dato maestro.

Los datos ingresados en la aplicación se sincronizan en el servidor web y, a partir de un navegador, se permite cruzar diferentes datos, con el propósito de analizar la correlación de información y su posición georreferenciada. Así, por ejemplo, podemos analizar existencias de una determinada enfermedad recurrente en un área geográfica, cruzándolas con datos ambientales que pueden ser útiles para obtener nuevas conclusiones y actuar consecuentemente.

Al momento de la redacción de este artículo, existe una versión de "prueba piloto" de la App + Salud, disponible para cualquier usuario en http://www.appsalud.unp.edu.ar compuesta por: a) una aplicación para sistema android 5.0 o superior, y b) un servidor web (Figura 4). Actualmente se están realizando, tanto en Argentina como en Brasil, diversos test de usabilidad y estabilidad de la pruebaconcepto on-line.

Tanto el colector y visualizador (aplicación para Android), como el sistema de persistencia (webserver) fueron desarrollados sobre frameworks que permiten un mantenimiento rápido, con el propósito de adaptar las nuevas necesidades del proyecto que van surgiendo de los test y para la próxima etapa de "implementación piloto". La aplicación para celulares o tablets Android permite recolectar datos sobre familia, individuo y vivienda, para posteriormente ser sincronizadas en el servidor web, donde se almacenan los datos. El sistema está desarrollado de modo de dar soporte al proyecto piloto. No obstante, todavía resta desarrollar su articulación con otros sistemas operativos como Iphone y, en función de los test, madurar errores y pruebas realizadas en el periodo actual. 


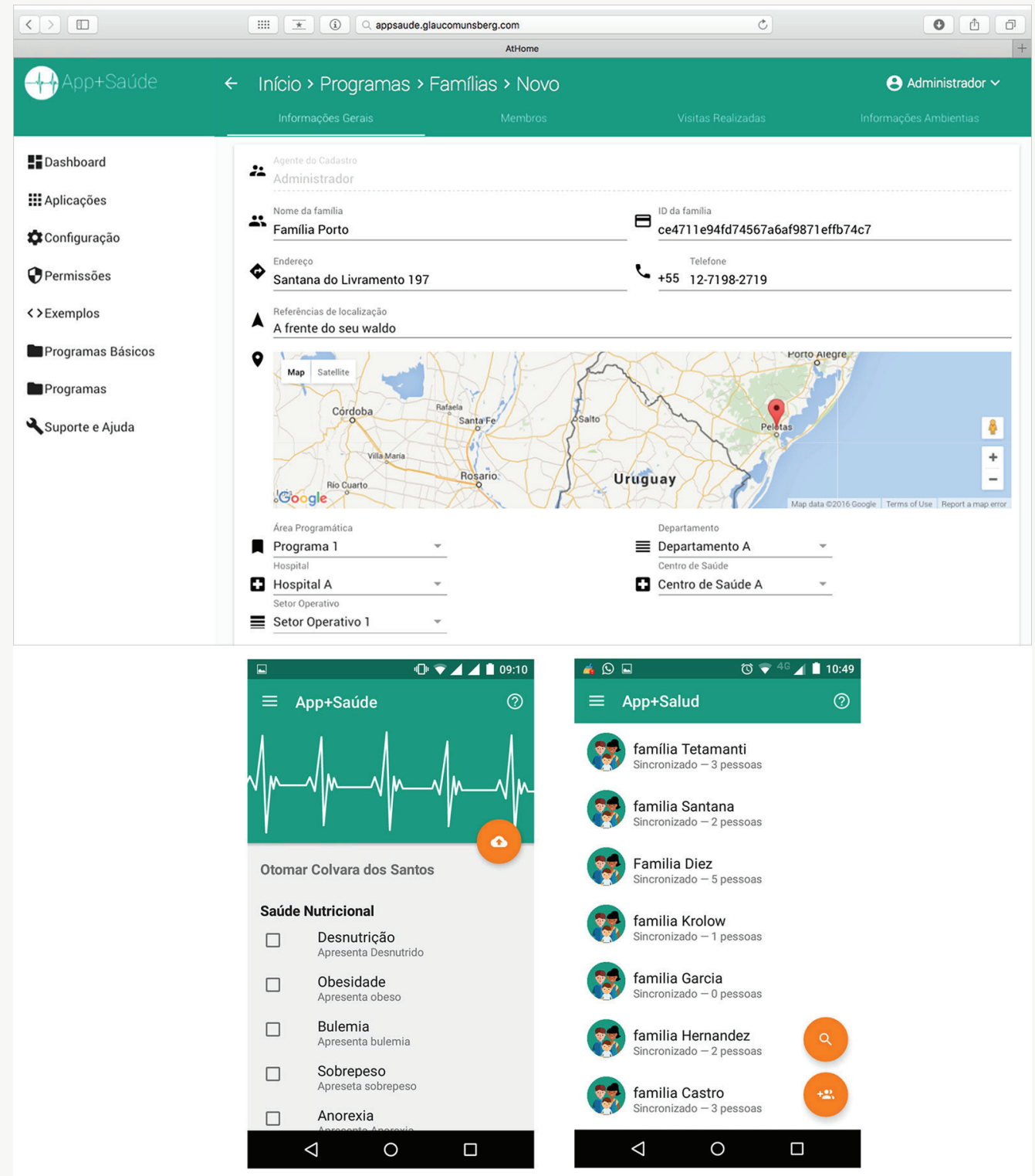

Figura 4. Vistas del sistema web y de la aplicación de App+Salud.

Fuente: Elaboración propia a partir de la aplicación App+Salud.

En el servidor web, es posible visualizar todos los datos recolectados por la aplicación, pero aun es necesario el desarrollo de programas internos que permitan, a partir de la interfaz del actual servidor, cruzar datos específicos sobre el mapeo de OpenStreetMap, permitiendo comprender la correlación entre la información cargada y las actualizaciones cartográficas.
En este sentido, el desarrollo de App + Salud prevé una articulación revolucionaria en el campo de la cooperación de los enfoques analítico y fenomenológico en geografía. La incorporación de un API (Application Programming Interface) de OpenStreetMap permite modificar los mapeos base, incorporando datos (en polígonos, líneas y puntos) con el agregado de 
contenido de la textura social. Esto transforma la propuesta en innovadora, ya que no se ajusta precisamente a los parámetros de la experticia en la carga de datos georreferenciados al mapa, sino que abre las puertas a un proceso de traducción permanente, a partir de mapeos sociales producidos mediante cartografía social con la participación de la población local involucrada. Así, por ejemplo, se plantea para la próxima etapa de implementación piloto, la realización de rondas de talleres de cartografía social en diferentes localidades, para la traducción de la información recopilada en OpenStreetMap, que es además una plataforma simplificada para la carga. De este modo, podrán incorporarse objetos con impronta de contenido social, de modo que forma y contenido no sean fijos, sino dinámicos como el propio espacio de interacción experiencial de los agentes de salud comunitaria. Esta interrelación de formas con contenido local en el mapa, más la información sanitaria agregada por el dispositivo, entendemos que enriquecerá profundamente el trabajo de los agentes de salud comunitaria en terreno, al tiempo que permitirá, según el nivel de escala de visualización y análisis, fortalecer las potencialidades de la prevención y reacción del sistema sanitario.

\section{DISCUSIÓN}

El análisis del proceso salud-enfermedad se ha visto enriquecido en los últimos años con la introducción del término territorio como variable que permite comprender en profundidad las necesidades de salud y su distribución en grupos humanos. Progresivamente, el concepto de salud y geografía fue migrando de paquetes informáticos que permitían adosar datos a mapas, a la utilización de datos que, contextualizados en zonas geográficas, permiten identificar "patrones de distribución de riesgos", paso ineludible en la búsqueda de clusters de inequidad y, por lo tanto, una poderosa herramienta en las definiciones de distribución de recursos sociales en salud ${ }^{(32,33)}$. Diversos cambios en el conocimiento y avances tecnológicos refinaron estos avances desarrollando una mixtura de metodologías que vinculan abordajes cualitativos y cuantitativos y que permiten, desde esta perspectiva, un avance sustancial, particular y dinámico en el conocimiento de cómo se suceden los fenómenos sanitarios en un colectivo en un territorio determinado.

Sin embargo, los adelantos tecnológicos y en el conocimiento no pudieron soslayar el enfoque hegemónico de las políticas de salud que, a pesar de contar con diagnósticos conducentes para un cambio socialmente positivo, en la práctica instrumentaron un tipo de racionalidad mecánica y segmentaria que fortalece las tramas institucionales hegemónicas de los servicios de salud, históricamente anodinas a las necesidades sociales en salud de una población y más orientadas al control social, un objetivo claramente incumplido ${ }^{(34)}$.

Pero fue tal el desarrollo de los sistemas de información geográfica y las técnicas de análisis geográficos, a veces en contraposición o complementariedad con las líneas de epidemiología crítica que suscriben a la geografía crítica y fenomenológica, que indujeron a modificar el concepto mismo de territorio, elevándolo a un concepto multidimensional que, a diferencia de otros conceptos muy populares en salud pública, recorrió el camino inverso: en vez de ser conceptualizado y luego operativizado, primero fue operativizado y posteriormente conceptualizado. La geosemántica, entonces, es fruto de la posibilidad de manejar datos masivos a los cuales se debe dar sentido y referencia con relación a un espacio geográfico; por ende, esta perspectiva tiene menos posibilidad de ser instrumental a la manutención de los arreglos institucionales vigentes y más orientados a mostrar las diferencias en grupos sociales tal y cual suceden. Este tipo de proyectos reorienta las fuerzas a un equilibrio entre el creciente interés en la gestión de sistemas de salud (una dimensión con un énfasis particular en los aspectos biopolíticos de las organizaciones de salud) con una mayor apertura a la búsqueda de coherencia entre las necesidades de salud 
de una población y las acciones destinadas a resolverlas ${ }^{(35,36)}$.

Así, la masificación de los smartphones y el uso cada vez más frecuente de mHealt, proporciona la posibilidad de articular los sistemas de información geográficos con dispositivos móviles en el territorio. Al mismo tiempo, la posibilidad de incorporar diversas capas de información que pemite, tanto visualizar como analizar los fenómenos en el territorio, se presenta como otro de los beneficios de estos sistemas.

El caso de la App+Salud se presenta como una alternativa inicial para la articulación de información social y dinámica, con datos históricos sociosanitarios de cada familia e integrados. Esta particularidad permitirá avanzar en análisis más profundos e integrados sobre la relación entre ambiente, sociedad y salud, tanto en áreas rurales como urbanas, como se propone desde la epidemiología crítica, en la que el dato duro no se encuentra escindido de la singularidad del sujeto y de lo colectivo.

La posibilidad de contar con datos nominalizados y georreferenciados es un avance más en la implementación efectiva de una cobertura universal de salud. Conocer al individuo, su entorno, sus principales datos sociosanitarios de interés y colocarlo en un contexto geográfico es un avance en la visibilización de sujetos en condiciones de inequidad socioeconómica y, por lo tanto, un paso más en la construcción de ciudadanos sujetos de derechos. La complejidad de los datos, así como su abultado volumen impedían la utilización de la información de manera conducente con este objetivo. Saltar esa barrera tecnológica también propondrá otros desafíos. Uno de ellos será saber de qué manera coordinarán las distintas disciplinas para lograr un conocimiento integrado y conducente, necesario para abordar las problemáticas sociales que se resisten a intervenciones mecanizadas y solo con una mirada disciplinar. El otro desafío es de qué manera aprovechará el sistema de salud las enormes ventajas que le brinda manejar gran volumen de datos con un nivel de complejidad inalcanzable hasta hace poco. Ese desafío, a nivel macro, será qué medidas se diseñarán con relación a la información que se genere $y$, a nivel micro, cómo los agentes de salud comunitaria desarrollarán habilidades relacionadas con la comunidad, aprovechando una sistematización de datos que los exceptúa de la generación de informes consolidados de carácter manual y que ocupan innecesariamente gran parte de su tiempo.

Este último punto, el desarrollo de habilidades y dispositivos relacionados a mejorar el desempeño de los trabajadores de salud, no es un dato menor. La evidencia muestra que no todas las intervenciones realizadas por trabajadores comunitarios de la salud son efectivas, y que su aporte a una mejora del nivel de salud de una comunidad se relaciona con cómo se orientan sus esfuerzos hacia poblaciones vulnerables con diferentes problemáticas y al rol que ocupan en equipos de salud sólidamente constituidos ${ }^{(37,38)}$. Es decir, para poder orientar o reorientar la dinámica de sus acciones, herramientas como la cartografía social y aplicaciones en dispositivos móviles orientadas a la sistematización de información impresionan ser una dupla prometedora en este sentido.

\section{CONCLUSIONES}

Generar un sistema de monitoreo georreferenciado e integral que facilite la gestión de la salud comunitaria, así como las tareas de prevención e investigación permanente en cuestión sanitaria, permite ampliar las posibilidades de atención y cobertura en salud, sobre todo, en áreas rurales y poblaciones vulnerables. La articulación de mapeos con contenidos sociales y datos cruzados levantados por los agentes de salud comunitaria permitirá, además, poder actuar con mayor eficacia, por ejemplo, en emergencias climáticas, visitando en primer lugar a aquellos individuos que lo requieren por determinadas causas de salud $\mathrm{o}$, en inversiones en infraestructura, a partir de la visualización de determinadas recurrencias en problemáticas sanitarias, asociadas con cuestiones ambientales. 
El desarrollo del proyecto App +Salud resulta una herramienta multidisciplinaria y binacional que genera un producto de innovación social, pues utiliza los más modernos elementos tecnológicos existentes y, al mismo tiempo, se caracteriza por la accesibilidad simple a bajos costos, mientras que brinda soluciones a un problema demandado por la comunidad sanitaria: la sistematización, articulación y georreferenciación de la información.

En el momento de cierre de este artículo, el grupo de trabajo ha finalizado la fase de prueba de la App + Salud, con agentes de salud comunitaria de Argentina y Brasil en diferentes puestos y centros de salud, prevista inicialmente hasta fines de 2016. Se estima que posteriormente se pueda avanzar con la experiencia de "implementación piloto" que prevé la puesta en marcha del sistema, en una zona geográfica acotada, para evaluar potencialidades y problemas, al tiempo que se realicen permanentes ajustes. En este marco, ya se encuentran en curso las acciones de convenio pertinentes con los ministerios de salud de Chubut y de Rio Grande do Sul, y los municipios de Comodoro Rivadavia y Pelotas.

Finalmente nos interesa rescatar la riqueza de enfoques que otorga el abordaje. A partir de la multidisciplina se ha podido avanzar desde una perspectiva fenomenológica, construyendo el método en el propio proceso $^{(39)} \sin$ por ello excluir la lógica de los nuevos paradigmas de la geotecnología ${ }^{(40)}$. Todo el camino recorrido refuerza la convicción de que, a partir de lo múltiple, es más factible avanzar en soluciones complejas, profundizando en la experiencia que supone compartir conocimientos y modos de hacer, con el propósito de generar soluciones producidas e implicadas con los propios sujetos que día a día y en territorio construyen el solidario camino de una salud comunitaria.

\section{AGRADECIMIENTOS}

Los proyectos en los cuales se encuadra este artículo se denominan "App+Saúde: Sistema georreferenciado e comunitário para a gestão, mobilidade e acessibilidade a saúde" financiado por el Conselho Nacional de Desenvolvimento Científico e Tecnológico (CNPq) (2014-2016) y "Accesibilidad a servicios públicos y políticas sociales en Patagonia Central" financiado por la Secretaría de Ciencia y Técnica de la Universidad Nacional de la Patagonia San Juan Bosco (20152018); además de los aportes a proyectos y becas financiadas por la Secretaría de Políticas Universitarias del Ministerio de Educación de la Nación Argentina, y el Consejo Nacional de Investigaciones Científicas y Técnicas (CONICET). A su vez, se ha contado con el apoyo financiero para la realización de diversas actividades de intercambio, trabajos de campo y movilidad, de la Secretaría de Extensión de la Universidad Nacional de la Patagonia San Juan Bosco (Argentina) y de la Facultade de Arquitetura de la Universidade Federal de Pelotas (Brasil). Se agradecen los aportes de Beatríz Escudero, directora del GIGAT, Susana Muñóz, directora de la Tecnicatura Superior en Salud Comunitaria sede Comodoro Rivadavia; Luis Avilés del Hospital Rural Río Mayo; María Ancaleo, del Puesto Sanitario Aldea Beleiro y Graciela Oliva del Hospital Rural de Río Senguer. También se agradecen los aportes metodológicos de Magali Chanampa, Alberto Vázquez y la colaboración en cartografía social de María de los Ángeles Jaimes, Pamela Gómez y Nadia Martínez.

\section{REFERENCIAS BIBLIOGRÁFICAS}

1. Schneider H, Okello D, Lehmann U. The global pendulum swing towards community health workers in low-and middle-income countries: a scoping review of trends, geographical distribution and programmatic orientations, 2005 to 2014. Human Resources for Health. 2016;14(1):65.

2. Starfield B. Equidad en salud y atención primaria. Gerencia y Políticas de Salud. 2001;1(1):7-16.

3. Diez Tetamanti JM, Escudero B. La construcción de un dispositivo de intervención a través de cartografía social. In: Diez Tetamanti JM, Escudero B. Cartografía social: investigación e intervención desde las ciencias sociales, métodos y experiencias de aplicación. Comodoro Rivadavia: Universitaria de la Patagonia; 2012. 
4. Montoya Arango V. El mapa de lo invisible: silencios y gramática del poder en la cartografía. Universitas Humanística. 2007; 63(63):155-179.

5. Guattari F, Rolnik S. Micropolítica: cartografias do desejo. 9a ed. Petrópolis: Vozes; 2008.

6. Passos E, Kastrup V, Escóssia L. Pistas do método da cartografia: pesquisa-intervenção e produção de subjetividade. Porto Alegre: Sulina; 2014.

7. Santos M. De la totalidad al lugar. Barcelona: Oikos-Tau; 1996.

8. Harley JB. The new nature of maps: essays in the history of cartography. Baltimore: Johns Hopkins University Press; 2001.

9. Tuan YF. Espaço e lugar: a perspectiva da experiência. São Paulo: Difel; 1983.

10. Kastrup V. Cartografiar é traçar um plano común. In: Pistas do método da cartografia: pesquisa-intervenção e produção de subjetividade. Porto Alegre: Sulina; 2014. p. 15-41.

11. Carballeda AJM. El territorio como relato: una aproximación conceptual. Revista Margen. 2015;76:1-6.

12. Breilh J. La epidemiología crítica: una nueva forma de mirar la salud en el espacio urbano. Salud Colectiva. 2010;6(1):83-101.

13. Spinelli $H$. Volver a pensar en salud: programas y territorios. Salud Colectiva. 2016;12(2):149-171.

14. Buzai GD. Análisis espacial de la salud: resoluciones con sistemas de información geográfica. Buenos Aires: Editorial Lugar; 2015.

15. Barcellos C, Ramalho WM, Gracie R, Magalhães MAFM, Fontes MP, Skaba DA. Georreferenciamento de dados de saúde na escala submunicipal: algumas experiências no Brasil. Epidemiologia e Serviços de Saúde. 2008;17(1):59-70.

16. Hino P, Villa TCS, Sassaki CM, Nogueira JA, Santos CB. Geoprocessing in health area. Revista Latino-Americana de Enfermagem. 2006;14(6): 939-943.

17. Müller EPL, Cubas MR, Bastos LC. Georreferenciamento como instrumento de gestão em unidade de saúde da família. Revista Brasileira de Enfermagem. 2010;63(6):978-982.

18. Da Fonseca JE, Do Carmo TA, Leme SM, Cordeiro RC, Antonio RD, Camolesi Junior L. Utilizando mapas dinâmicos georeferenciados para caracterizar o território do PSF [Internet]. Piracicaba: 7a Mostra Académica Universidade Metodista de Piracicaba. [citado 16 oct 2016]. Disponible en: https://tinyurl.com/y8d9judr.

19. CommCare: mobile data collection platform [Internet]. Cambridge: Dimagi Inc. 2010 [citado 3 oct 2016]. Disponible en: https://www.commcarehq.org/home/.

20. Macueve G. E-health and m-health in Mozambique: innovations likely to lead to increased retention and performance of Community Based Agents (CBAs) [Internet]. Maputo; 2010 [citado 4 jun 2017]. Disponible en: https://tinyurl.com/ yct2tr9b.

21. De Tolly K, Alexander H. Soul Beat Africa: HIV-AIDS. Innovative use of cellphone technology for HIV/AIDS behaviour change communications: 3 pilot projects [Internet]. 2009 [citado 4 jun 2017]. Disponible en: https://tinyurl.com/ yae7v6h2.

22. United Nations Development Program Mobile technologies and empowerment: enhancing human development through participation and innovation [Internet]. 2012 [citado 4 jun 2017]. Disponible en: https://tinyurl.com/cyfpo8y.

23. Diel L. El concepto de diferencia en Gilles Deleuze como propuesta crítica ante la ontología tradicional aristotélica. A Parte Rei Revista de Filosofía. 2011;75:1-5.

24. Facultad de Humanidades y Ciencias Sociales, Universidad Nacional de la Patagonia San Juan Bosco. Ensayos de Cartografía Social en Patagonia [Internet]. Comodoro Rivadavia. 2014 [citado 20 oct 2016]. Disponible en: https://tinyurl.com/ y87brzjt.

25. Vázquez A, Escudero B. Exploración metodológica: territorialidades y acceso a la atención de la salud en localidades patagónicas. In: Diez Tetamanti JM. Hacia una geografía comunitaria. Comodoro Rivadavia: Universitaria de la Patagonia EDUPA; 2014. p. 45-62.

26. Chanampa M, Diez Tetamanti JM, Duarte $Y$, Jaimes M, Martínez N, Gómez P. Accesibilidad a la salud y estrategias de movilidad: caso aldea Beleiro. Revista de Informes Científicos y Técnicos. 2015;1(1):54-77.

27. Vázquez A, Massera C. Repensando la geografía aplicada a partir de la cartografía social. In: Diez Tetamanti JM, Escudero B. Cartografía social: investigación e intervención desde las ciencias sociales, métodos y experiencias de aplicación. Comodoro Rivadavia: Universitaria de la Patagonia; 2012. 
28. Moreno JL. Sociodrama. Buenos Aires: Lúmen; 1983.

29. Carballeda AJM. La intervención en lo social: exclusión e integración en los nuevos escenarios sociales. Buenos Aires: Paidós; 2012. p. 75-94.

30. Carballeda AJM. La intervención en lo social como dispositivo: una mirada desde los escenarios actuales. Trabajo Social UNAM. 2010;1:46-59.

31. Andrade Calderón MC, Muñoz Dagua C. El taller crítico: una propuesta de trabajo interactivo. Tabula Rasa. 2004;2:251-262.

32. Schneider MC, Castillo-Salgado C, Bacallao J, Loyola E, Mujica OJ, Vidaurre M, Roca A. Métodos de medición de las desigualdades de salud. Revista Panamericana de Salud Pública. 2002;12(6):398-414.

33. Organización Panamericana de la Salud. Uso de sistemas de informacion geografica en epidemiologia [Internet]. Boletín Epidemiológico. 1997;17(1) [citado 20 oct 2016]. Disponible en: https://tinyurl.com/y7bs48r2.

34. Loyola E, Castillo-Salgado C, Nájera-Aguilar $P$, Vidaurre $M$, Mujica OJ, Martínez-Piedra R. Los sistemas de información geográfica como he- rramienta para monitorear las desigualdades de salud. Revista Panamericana de Salud Pública. 2002;(6):415-428.

35. Chiara M. Territorio, políticas públicas y salud: hacia la construcción de un enfoque multidimensional para la investigación. Gerencia y Políticas de Salud. 2016;15(30):10-22.

36. Rodríguez C, Lamothe L, Barten F, Haggerty J. Gobernanza y salud: significado y aplicaciones en América Latina. Revista de Salud Pública. 2010;12(1):151-159.

37. Báscolo E. Gobernanza de las organizaciones de salud basados en Atención Primaria de Salud. Revista de Salud Pública. 2010;12(1):8-27.

38. Labonté R. Health systems governance for health equity: critical reflections. Revista de Salud Pública. 2010;12(1):62-76.

39. Careri F. Walkscapes ten years after. Revista de Estudios Urbanos y Ciencias Sociales. 2014;4(1):207-213.

40. Buzai G. D. Paradigma geotecnológico, geografía global y cibergeografía, la gran explosión de un universo digital en expansión. GeoFocus. $2001 ; 1: 24-48$. 